\title{
Panax notoginseng Saponins Regulate Macrophage Polarization under Hyperglycemic Condition via NF- $\kappa$ B Signaling Pathway
}

\author{
Yan Zhao, Jianlei Zheng $(\mathbb{D}$, Yongmei Yu, and Lihong Wang \\ Department of Cardiology, Zhejiang Provincial People’s Hospital, Hangzhou, Zhejiang 310014, China \\ Correspondence should be addressed to Jianlei Zheng; zhengjianlei@zjheart.com
}

Received 26 March 2018; Revised 10 June 2018; Accepted 11 July 2018; Published 30 July 2018

Academic Editor: Gernot Zissel

Copyright (c) 2018 Yan Zhao et al. This is an open access article distributed under the Creative Commons Attribution License, which permits unrestricted use, distribution, and reproduction in any medium, provided the original work is properly cited.

\begin{abstract}
Panax notoginseng saponins (PNS), the principal constituents derived from Panax notoginseng, have been extensively used for treating cardiocerebral vascular diseases in China and other Asian countries. The main effects of PNS were anti-inflammatory properties, inhibition of platelet aggregation, improvement of blood flow and insulin resistance, and so on. This study was carried out to explore the effects of PNS on macrophage polarization under hyperglycemic conditions. Human acute monocyte leukemia cell line THP-1 cells were induced into macrophages with Phorbol ester (PMA). Macrophages were then divided into five groups as follows: control ( $5.5 \mathrm{mMol} / \mathrm{l}$ glucose), hyperglycemia group ( $15 \mathrm{mMol} / \mathrm{l}$ glucose), hyperglycemia plus low-dose PNS (20ug/ml), hyperglycemia plus moderate-dose PNS (40ug/ml), and hyperglycemia plus high-dose PNS (60ug/ml). After 48hour cell culture, the percentages of M1- and M2-polarized macrophages were measured by flow cytometry analysis. Reverse transcription quantitative real-time polymerase chain reaction (RT-qPCR) was used to evaluate the Yml and arginase 1 expression in macrophages. Protein expression of arginase $1, \mathrm{NF}-\kappa \mathrm{B}$ p50, p65, and inhibitor of $\kappa \mathrm{B}(\mathrm{I} \kappa \mathrm{B})$ alpha phosphorylation in macrophages was identified with Western blotting. PNS, especially the high-dose PNS, remarkably increased M2 phenotype ratio in macrophages cultured with hyperglycemia, and the mRNA expression of $\mathrm{Yml}$ and arginase 1 in macrophages was also upregulated. Meanwhile, PNS remarkably increased the protein expression of arginase 1 and decreased $\mathrm{I} \kappa \mathrm{B}$-alpha phosphorylation and subunits of NF- $\kappa \mathrm{B}$ p50 and p65 from macrophages in culture medium with hyperglycemia. Taken together, our work demonstrated that PNS promote macrophages to transform M2 phenotype under hyperglycemic conditions through downregulating NF- $\kappa$ B signaling pathway.
\end{abstract}

\section{Introduction}

Atherosclerosis is regarded as a chronic inflammatory disease, and macrophages play a pivotal role in atherosclerosis from fatty streaks to plaque rupture [1]. Diversity and plasticity are hallmarks for macrophages, and recently substantial evidence demonstrated that plaque pathogenesis and evolution are influenced by macrophage activation and polarization [2]. Macrophages were grossly divided into two main phenotypes, proinflammatory M1 cells (classically activated macrophages) and anti-inflammatory M2 cells (alternatively activated macrophages). Different from phenotypic macrophages with corresponding markers, CD16 is a marker for M1-polarized macrophages, and M2-polarized macrophages are characterized by markers, such as CD206, Yml, and arginase $1[3,4]$. M2 phenotype cells are thought to have an important role in atherosclerotic plaques regression
[5]. It is mentioned that macrophage polarization is influenced by microenvironment including microbial products and cytokines [6-8]. Hyperglycemia has long been speculated to account for some effects of diabetes on cardiovascular complications caused by atherosclerosis [9]. It is well known that hyperglycemia significantly aggravates inflammatory response and oxidative stress. Importantly, previous studies showed that the phenotype of M1/M2 macrophages was imbalance and macrophages were more inclined to switch to $\mathrm{M} 1$ phenotypic polarization under hyperglycemic environment $[9,10]$.

PNS are the major effective ingredients extracted from Panax notoginseng. Numerous studies showed that PNS displayed significant antiatherogenic effects, including the abilities to limit the proliferation of vascular smooth muscle cells, reduce thrombosis risk, protect against artery injury, reduce inflammatory response, and ameliorate hyperglycemia and 
TABle 1: Primer sequences for quantitative real-time PCR.

\begin{tabular}{lccc}
\hline Gene & Primer & Sequences $\left(5^{\prime}-3^{\prime}\right)$ & Product size \\
\hline \multirow{2}{*}{ Homo $\beta$-actin } & Forward & $5^{\prime}$-AGCGAGCATCCCCCAAAGTT-3 & $285 \mathrm{bp}$ \\
Homo Yml & Reverse & $5^{\prime}$-GGGCACGAAGGCTCATCATT-3' & \\
& Forward & $5^{\prime}$-CCTTGACCGCTTCCTCTGTA-3' & $166 \mathrm{bp}$ \\
Homo arginase 1 & Reverse & $5^{\prime}$-GTTCCATCCTCCGACAGACA-3' & $210 \mathrm{bp}$ \\
& Forward & $5^{\prime}$-TGGACCCATCTTTCACACCA-3 & \\
\hline
\end{tabular}

insulin resistance [11-14]. However, little evidence has been elucidated on the relationship between PNS and macrophage polarization. The present studies were designed to address the effects of PNS on macrophage polarization under hyperglycemic conditions and to explore the relevant molecular mechanism.

\section{Materials and Methods}

2.1. Reagents and Chemicals. THP-1 was purchased from Shanghai Institutes for Biological Sciences, Chinese Academy of Sciences (Shanghai, China). Panax notoginseng saponins were purchased from Kunming Pharmaceutical Company (KPC) (Yunnan Province, China), which mainly contained notoginsenoside R1 9\%, ginsenoside Rg1 32\%, ginsenoside Re 5\%, ginsenoside Rb1 36\%, and ginsenoside Rd 8\%. The chemical purity of PNS was about $90 \%$. RPMI-1640 medium without glucose was bought from Gibco (Carlsbad, CA). PMA, D-glucose, and NF- $\kappa$ B inhibitor of BAY 11-7082 were from Sigma-Aldrich (St. Louis, MO, USA). M-MLV Reverse Transcriptase was from GeneCopoeia (Maryland, USA). SYBP Premix Ex Taq TM was from Takara (Shiga, Japan). TRIzol, RIPA, and BCA protein assay kit were from Thermo Fisher Scientific (Waltham, USA). Real-time PCR detection system was from Bio-Rad (Hercules, CA, USA). Flow cytometry was purchased from BD Biosciences (CA, USA). Rabbit polyclonal p50 and p65 antibodies were from Proteintech (Chicago, USA). Mouse monoclonal antibodies to CD16 and CD206 and rabbit monoclonal antibodies to phospho- $\mathrm{I} \kappa \mathrm{B}$ alpha and arginase 1 were from Abcam (Cambridge, UK). Mouse monoclonal antibody to $\beta$-actin and rabbit polyclonal antibody to $\beta$-tubulin were purchased from Boster Biological Technology Co., Ltd. (Wuhan, China) and Cell Signaling Technology (Danvers, MA). ECL kit was purchased from Pierce Biotechnology (Waltham, USA).

2.2. Cell Culture and Treatment. The human THP-1 cell, an acute monocytic leukemia cell line, was cultured in RPMI 1640 media supplemented with $10 \%$ fetal bovine serum (FBS), 100 units $/ \mathrm{mL}$ penicillin, and $100 \mathrm{ug} / \mathrm{ml}$ streptomycin at $37^{\circ} \mathrm{C}$ in a $5 \% \mathrm{CO}_{2}$ incubator. THP-1 cells seeded in 6well culture plates at a density of $5 \times 10^{5}$ cell $/ \mathrm{ml}$ with $200 \mathrm{nM}$ PMA for $48 \mathrm{~h}$ were triggered to undergo differentiation into macrophages. Then, nonattached cells were removed by aspiration and adherent cells were washed three times with PBS. Macrophages were then divided into control $(5.5 \mathrm{mMol} / 1$ glucose), hyperglycemic group ( $15 \mathrm{mMol} / \mathrm{l}$ glucose), hyperglycemia plus different-dose PNS groups $(20 \mathrm{ug} / \mathrm{ml}, 40 \mathrm{ug} / \mathrm{ml}$, and $60 \mathrm{ug} / \mathrm{ml}$, resp.) and cultured in the corresponding environment for $48 \mathrm{~h}$. The THP-1 macrophages were exposed to $5 \mathrm{uMol} / \mathrm{l} \mathrm{NF}-\kappa \mathrm{B}$ inhibitor BAY $11-7082$ for 2 hours before they were stimulated with hyperglycemia for 24 hours. The experiments were repeated three times independently.

2.3. Flow Cytometry. Cultured cells were washed with cold PBS and incubated with CD206 antibody conjugated with $\mathrm{PE}$ and CD16 antibody conjugated with FITC at $4^{\circ} \mathrm{C}$ for $30 \mathrm{~min}$. The cells were centrifuged at 12000 for $2 \mathrm{~min}$ at $4^{\circ} \mathrm{C}$ and resuspended in PBS. The expressions of CD16 and CD206 were determined by flow cytometry to identify the macrophage polarization phenotype with a BD Biosciences Digital LSR II. Anti-CD16 and anti-CD206 antibodies were from Abcam (Cambridge, UK, dilution 1:50 and 1:100, resp.); data were analyzed using FlowJo software (Tree Star, Inc.).

\subsection{RNA Isolation and Quantitative Real-Time Polymerase} Chain Reaction. The total RNA was extracted from macrophages with TRIzol Reagent, and the concentration was measured at $260 \mathrm{~nm} / 280 \mathrm{~nm}$ absorbance ratio. The total RNA was reverse-transcribed to cDNA using a high-capacity cDNA Reverse Transcription Kit. Real-time PCR was carried out with SYBP Premix Ex Taq TM following the manufacturer's instructions. Samples for RT-qPCR test were repeated in triplicate. The PCR was performed on a real-time PCR detection system (Bio-Rad) as follows: $50^{\circ} \mathrm{C}$ for $2 \mathrm{~min}$ and $95^{\circ} \mathrm{C}, 10 \mathrm{~min}$, followed by 40 cycles of denaturation at $95^{\circ} \mathrm{C}$ for $30 \mathrm{~s}$ and annealing at $60^{\circ} \mathrm{C}$ for $30 \mathrm{~s}$. Semilog amplification curve was analyzed using the $2^{-\triangle \Delta \mathrm{Ct}}$ method, and the relative gene expression was normalized to that of $\beta$-actin used as an endogenous control. The PCR primer sequences are listed in Table 1.

2.5. Protein Extraction and Western Blotting. Macrophages were lysed using Radio-Immunoprecipitation Assay (RIPA) lysis buffer at $4^{\circ} \mathrm{C}$ for $30 \mathrm{~min}$ and subjected to $12000 \mathrm{rpm}$ centrifugation at $4^{\circ} \mathrm{C}$ for $5 \mathrm{~min}$. The protein concentration was measured by BCA protein assay kit. The lysates were separated by $12 \%$ sodium dodecyl sulfate-polyacrylamide gel electrophoresis (SDS-PAGE), transferred to polyvinylidene fluoride (PVDF) membranes, and blocked with 5\% skim milk. The blots were analyzed with antibodies according to the manufacturer's instructions and visualized by peroxidase and an enhanced chemiluminescence system. The corresponding antibodies were used in the present study including anti-p50 and anti-p65 (1:1000 dilution and 1:2000 
dilution, resp.; Proteintech, USA), anti-arginase 1 and antiphospho-I $\kappa \mathrm{B}$ alpha (1:1000 dilution and 1:10000 dilution, resp.; Cambridge, UK), anti- $\beta$-actin (1:200 dilution; Boster Biological Technology Co., Ltd., China), and anti- $\beta$-tubulin (1:1000 dilution; Danvers, MA).

2.6. Statistical Analysis. Data analysis was performed with SPSS 13.0 software. Data were expressed as mean \pm standard deviation. Comparisons between two groups were evaluated by $t$-test. A $p$ value less than 0.05 was considered statistically significant.

\section{Results}

3.1. PNS Regulate Macrophage Plasticity towards the M2Polarized Phenotype under Hyperglycemic Conditions. To explore the correlation between PNS treatment and macrophage polarization, we analyzed the percentages of macrophage phenotype under hyperglycemic conditions treated with different-dose PNS. CD16 and CD206 were used as biological markers for M1 and M2 macrophages, respectively. Our findings indicated that PNS dosedependently inhibited the M1 macrophage polarization and promoted the macrophages towards M2-polarized phenotype under hyperglycemic conditions after 48-hour culture according to the results of flow cytometry analysis (Figures $1(\mathrm{a})-1(\mathrm{~d}))$. The ratio of M1/M2 decreased with the increasing concentrations of PNS, which was shown in Figure 1(e). These data suggested that PNS may switch the macrophages under hyperglycemic conditions from proinflammatory M1 cells towards an anti-inflammatory M2 phenotype, and the ratio of M1/M2 was changed under hyperglycemic environment with PNS in a dose-independent manner.

\subsection{The Effect of PNS on Gene Expression of Ym1 and Arginase} 1 in Macrophages. The gene levels of Yml and arginase 1 are important biomarkers for M2 phenotypic macrophages. Real-time PCR results showed that the mRNA expression levels of Yml and arginase 1 were lower in hyperglycemic group than in control group $(p<0.01)$ (Figures 2 (a) and $2(b))$. However, these two genes' expression increased in the PNS-treated groups. The levels of Yml and arginase 1 in macrophages with moderate- and high-dose PNS treatment were remarkably higher compared with hyperglycemic group $(p<0.01)$. The above results demonstrated that PNS regulated macrophage plasticity towards an M2-specified phenotype through influencing the genes expression of Yml and arginase 1 under hyperglycemic conditions. We further demonstrated that arginase 1 protein expression was markedly increased under hyperglycemic environment with moderate- and highdose PNS treatment (Figures 2(c) and 2(d)).

3.3. PNS Influence NF- $\kappa B$ Signaling Pathway Regulating M2Polarized Macrophages Expression. NF- $\kappa \mathrm{B}$ signaling pathway plays a pivotal role in inflammation response and macrophage polarization. To explore whether PNS regulate THP-1 derived macrophage polarization through NF- $\kappa \mathrm{B}$ signaling pathway under hyperglycemic conditions, subunits p50 and p65 of NF- $\kappa \mathrm{B}$ were examined by Western blotting analysis. We found that p50 and p65 expressions in hyperglycemia group were remarkably higher than those in control group $(p<0.01)$ (Figure 3$)$. However, the expression of NF$\kappa \mathrm{B}$ p50 and p65 remarkably decreased after moderate- and high-dose PNS treatment $(p<0.01)$. Considering the highdose PNS with the strongest effect on prompting M2 type macrophage polarization and lowering NF- $\kappa$ B subunits p 65 and p50 expression, we further tested the effect of highdose PNS on I $\kappa \mathrm{B}$-alpha phosphorylation. As a result, our study showed that $5 \mathrm{uM}$ NF- $\kappa$ B inhibitor BAY 11-7082 and high-dose PNS significantly decreased the expression of $\mathrm{I} \kappa \mathrm{B}$ alpha phosphorylation, compared with hyperglycemia group $(p<0.01)$ (Figures 4(a) and 4(b)). Meanwhile, the expression of arginase 1 was slightly higher in group with $\mathrm{NF}-\kappa \mathrm{B}$ inhibitor pretreatment than in hyperglycemia group $(p<0.05)$ (Figures 4(c) and 4(d)).

\section{Discussion}

Atherosclerosis is considered as a chronic inflammatory disease, and macrophages play a critical role in the initiation and development of atherosclerosis. In addition to adjusting cholesterol metabolism, forming foam cell, and secreting matrix metalloproteinases and cytokines, it is noted that macrophage polarization under different microenvironment was involved in the pathological process of atherosclerosis [15]. Panax notoginseng saponins (PNS) are the major effective ingredients extracted from Panax notoginseng, which have been extensively used for treating cardiocerebral vascular diseases in China and other Asian countries. In this study, we demonstrated that PNS promoted the macrophages to M2-polarized phenotype under hyperglycemic conditions via downregulating NF- $\kappa \mathrm{B}$ signaling pathway.

Plasticity and diversity are hallmarks of macrophages, and a continuum of proinflammatory and anti-inflammatory macrophages mainly including M1 and M2 were known as extreme polarization. M1 and M2 were also called classically and alternatively activated macrophages. Although both M1 and M2 macrophages existed in human atherosclerotic lesions, M1 macrophages are the dominant phenotype linked to plaque progression [16]. The macrophage polarization was a complicated dynamic response to different stimuli, and it is generally accepted that the tissue microenvironment regulates macrophage phenotypic polarization [17].

Cardiovascular diseases are major complications in type 1 and type 2 diabetes. Hyperglycemia may be an important factor associated with cardiovascular complications in diabetic patients [18]. Inflammation and lipid metabolism disorder are more intensive in diabetic patients than in nondiabetic population, which was related to sustained hyperglycemia, aggravating oxygen stress, and formation of advanced glycation end-products (AGEs) [19]. High glucose acts directly or indirectly via the generation of AGEs and reactive oxygen species on endothelial cells, smooth muscle cells, and macrophages [20]. AGE-modified albumin can inhibit SR-B1-mediated efflux of cholesterol to HDL [21]. Hyperglycemic state promotes the macrophages to adhere 

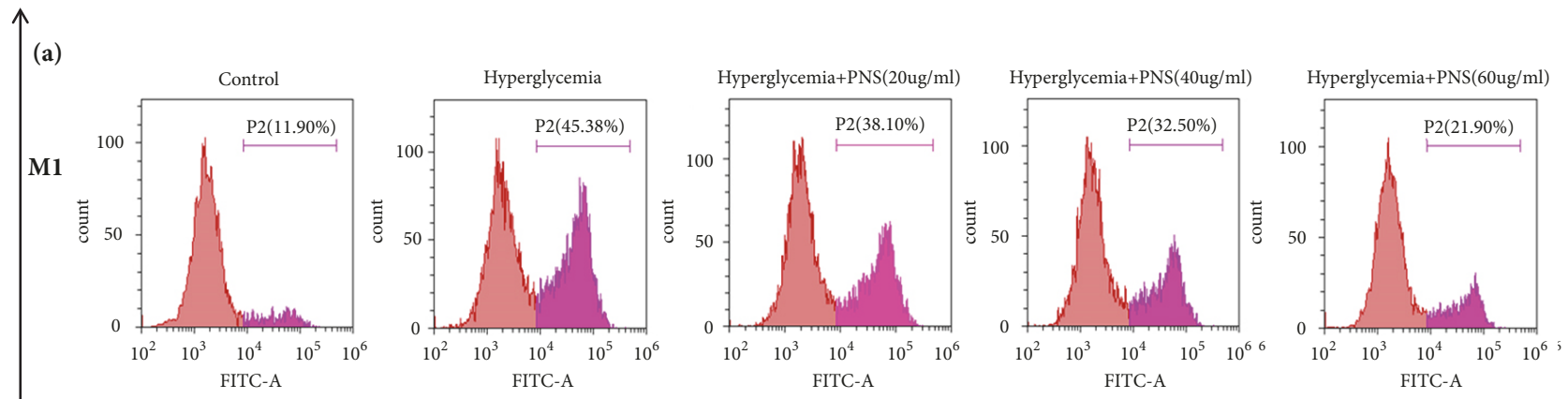

(b)
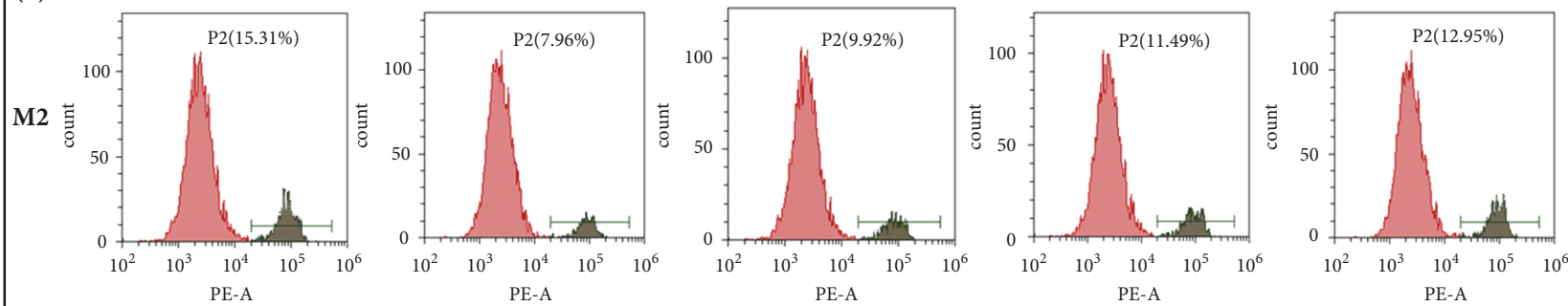

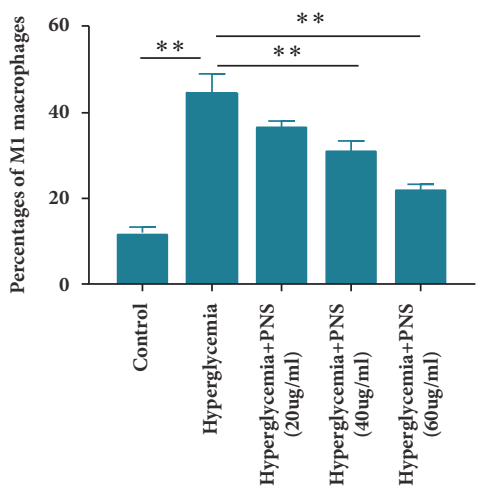

(c)

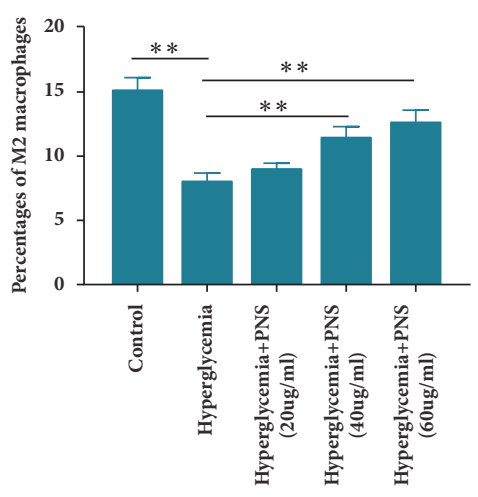

(d)

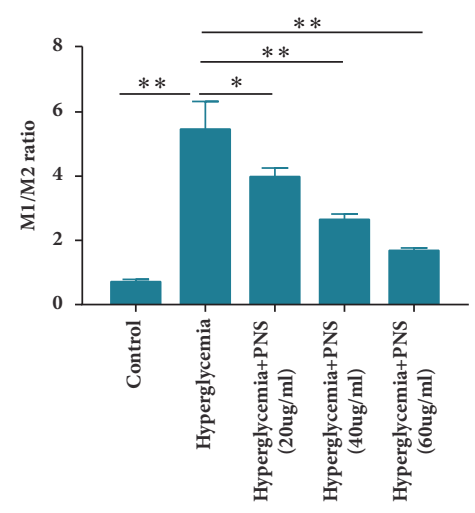

(e)

FIgURE 1: PNS treatment influences the differentiation of M1 and M2 macrophages under hyperglycemic conditions. The percentages of M1 and M2 phenotype macrophages under hyperglycemic conditions treated with different levels of PNS were measured according to flow cytometry analysis $(\mathrm{a}-\mathrm{d})$. The ratio of M1/M2 phenotype under hyperglycemic conditions treated with different levels of PNS was evaluated (e). CD16 as a biomarker for M1 phenotype macrophages conjugated with FITC and CD206 as a biomarker for M2 phenotype macrophages conjugated with PE, respectively. Data are presented as mean $\pm \mathrm{SE}$ ( $\mathrm{n}=3$ per group). $* *$ indicates $p<0.01$ and $*$ indicates $p<0.05$.

and transmigrate into the subendothelial space. In addition, hyperglycemia can prevent macrophages from converting into M2 phenotype, and AGEs also play an important role in adjusting the macrophages to form M1-polarized phenotype $[9,10,22,23]$.

Of the various Traditional Chinese Medicines, PNS are one of the most commonly applied products for treating cardiocerebral diseases due to their various beneficial effects. The main pharmacotherapeutic effects of PNS were proven to limit the proliferation of vascular smooth muscle cells, promote blood circulation, improve lipid and glucose metabolism, and inhibit inflammation responses and oxidative stress [11-14, 24, 25]. PNS adjust glucose metabolism by increasing glucose transporter 4 (GLUT4) expression and glycogen synthesis in 3T3-L1 adipocytes [26]. Several studies showed that PNS exert the anti-inflammatory effects on adjusting NF- $\kappa \mathrm{B}$ expression, activation of pregnane $\mathrm{X}$ receptor, and inhibition of toll-like receptor [13, 27, 28]. However, whether the PNS influence the macrophage polarization is still unknown.

In the current study, we demonstrated that PNS can promote the Yml and arginase 1 gene expressions in macrophages under hyperglycemic conditions and in a PNS concentrationdependent manner. It is reported that $\mathrm{Yml}$ and arginase 1 expression were higher in M2-polarized macrophages than those in M1-polarized macrophages. In the current study, we demonstrated that PNS can promote the Yml and arginase 1 gene expression in macrophages under hyperglycemic 


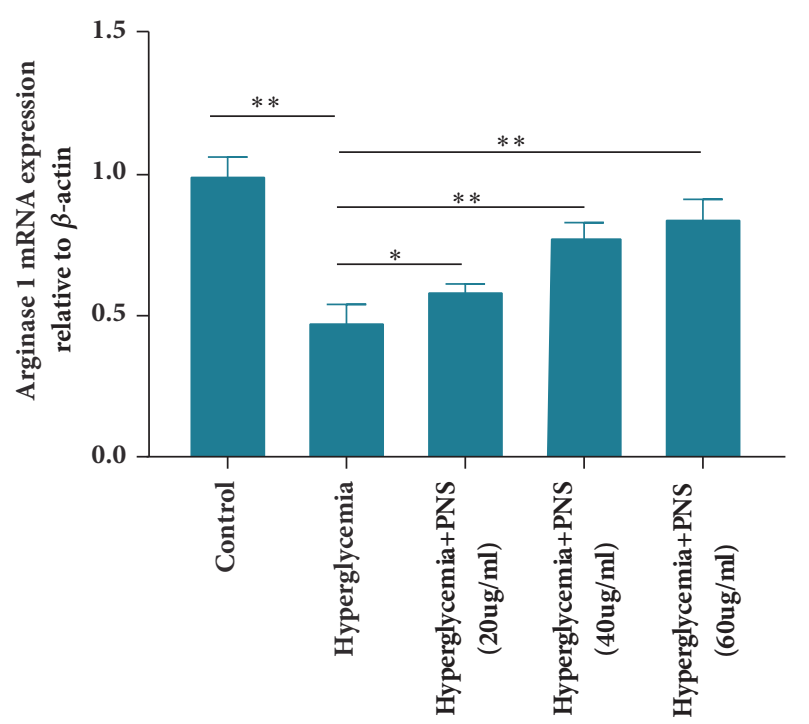

(a)

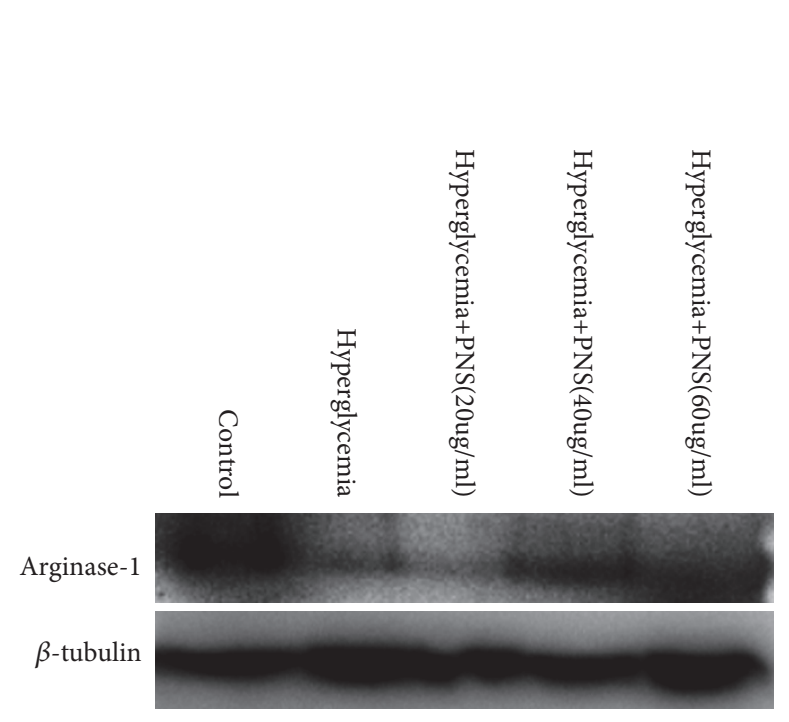

(c)

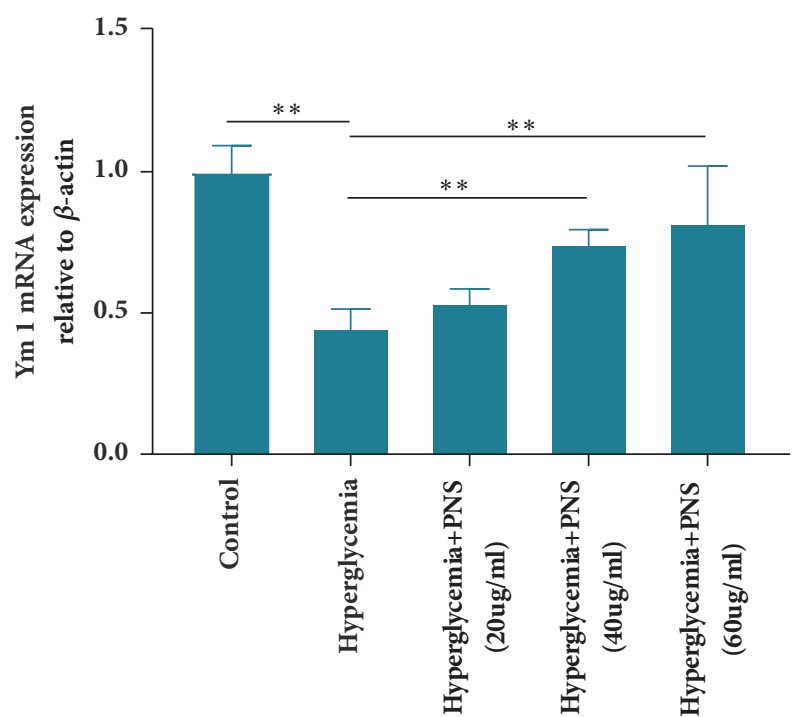

(b)

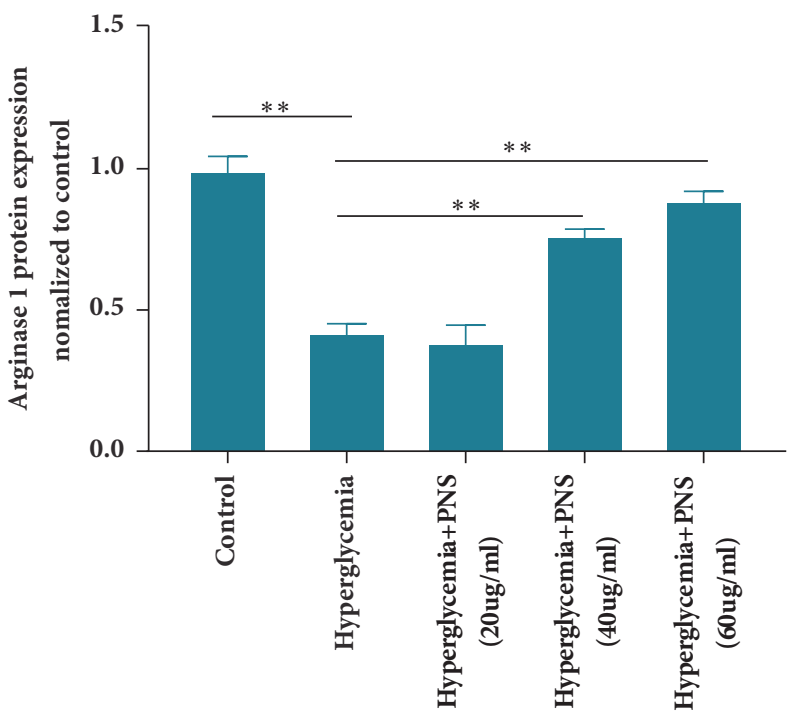

(d)

FIGURE 2: The effects of PNS treatment on gene expression of Yml and arginase I in macrophages under hyperglycemic state. The effects of different levels of PNS on gene expression of Ym1 and arginase 1 in macrophages under hyperglycemic state were assessed by RT-qPCR (a, b) ( $\mathrm{n}=5$ per group). Protein expression of arginase 1 in macrophages under hyperglycemic conditions at different concentration PNS treatment was examined by Western blot (c, d) ( $\mathrm{n}=3$ per group). Results are expressed as the mean \pm SE. $* *$ indicates $p<0.01$ and $*$ indicates $p<0.05$.

conditions and in a PNS concentration-dependent manner, which can reflect that PNS induced macrophages to turn into M 2 phenotype. The percentages of M2 phenotype macrophages were increased under hyperglycemic conditions with PNS treatment, which was proven by flow cytometry analysis according to the biomarkers expressed in different polarized macrophages. In addition, we further proved that PNS refrained the production of the NF- $\kappa \mathrm{B}$ p50 and p65 subunits and $\mathrm{I} \kappa \mathrm{B}$-alpha phosphorylation in macrophages cultured with hyperglycemia. In fact, NF- $\kappa$ B is a key transcription factor involved in various pathological processes such as macrophage polarization $[29,30]$. In quiescent cells, NF- $\kappa \mathrm{B}$ is mainly located in cytoplasmic environment due to the binding of a dedicated set of inhibitory proteins comprising the $\mathrm{I} \kappa \mathrm{B}$ family. Upon stimulation, $\mathrm{I} \kappa \mathrm{B}$ is phosphorylated by activated $\mathrm{I} \kappa \mathrm{B}$ kinase (IKK). The phosphorylation of $\mathrm{I} \kappa \mathrm{B}$ causes NF- $\kappa \mathrm{B}$, which can actively shuttle between the nucleus and cytosol, to enter nuclear region and induce gene expression [31]. According to the above findings, we speculated that PNS may promote the expression of M2 type macrophage via decreasing the level of NF- $\kappa \mathrm{B}$ signaling pathway mediated by $\mathrm{I} \kappa \mathrm{B}$-alpha phosphorylation.

In conclusion, PNS as a Chinese patent medicine for coronary artery disease mainly depend on their effects of antiinflammation, improving lipid and glucose metabolism. Our work found that anti-inflammation effect of PNS may partly 


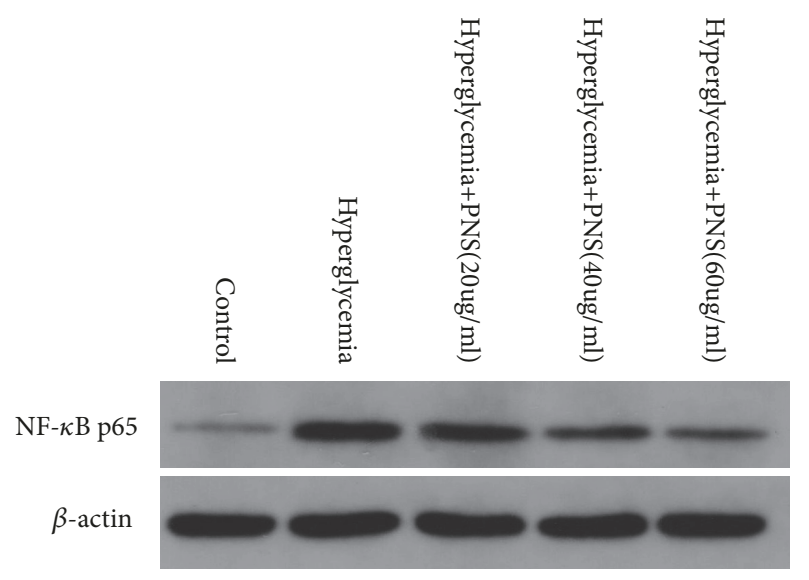

(a)

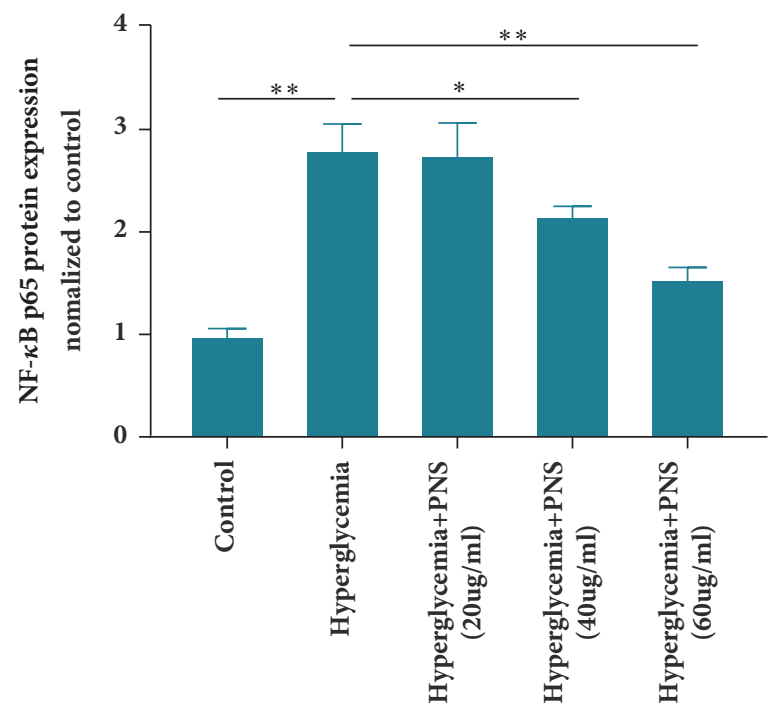

(c)

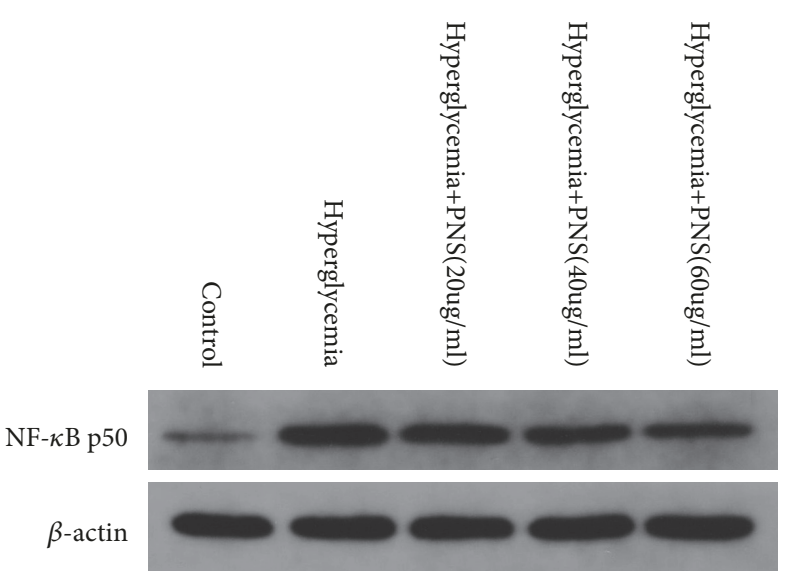

(b)

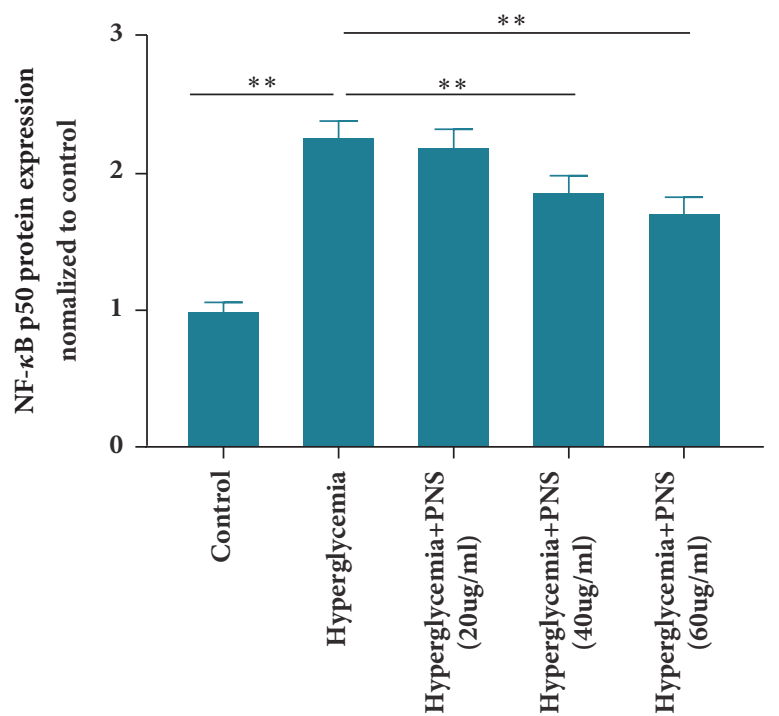

(d)

FIgURE 3: PNS promote M2-polarized macrophages expression by inhibiting NF- $\kappa$ B signaling pathway. The protein levels of NF- $\kappa \mathrm{B}$ p65 $(\mathrm{a}, \mathrm{c})$ and $\mathrm{p} 50(\mathrm{~b}, \mathrm{~d})$ in macrophages under hyperglycemic conditions treated with PNS were examined by Western blotting ( $\mathrm{n}=3$ per group). Results are expressed as the mean \pm SE. $* *$ indicates $p<0.01$.

be attributed to the fact that PNS induced macrophages into M2 phenotype, and NF- $\kappa$ B signaling pathway was involved in the macrophage polarization mediated by PNS. However, it is unclear whether the macrophage polarization caused by PNS affects the development of atherosclerosis in vivo. Animal experiments may clarify this mechanism in the following studies.

\section{Abbreviations}

AGEs: Advanced glycation end-products

GLUT4: Glucose transporter 4

I $\kappa$ B: $\quad$ Inhibitor of $\kappa \mathrm{B}$

KPC: $\quad$ Kunming Pharmaceutical Company

NF- $\kappa$ B: $\quad$ Nuclear factor-kappa B

PBS: $\quad$ Phosphate-buffered saline

PNS: $\quad$ Panax notoginseng saponins

RT-qPCR: Reverse transcription quantitative real-time polymerase chain reaction
SR-B1: Scavenger receptor class B1

THP-1: Human acute monocyte leukemia cell line.

\section{Data Availability}

The data used to support the findings of this study are available from the corresponding author upon request.

\section{Conflicts of Interest}

The authors declare that there are no conflicts of interest.

\section{Authors' Contributions}

Yan Zhao and Jianlei Zheng contributed equally to this work and are co-first authors. 


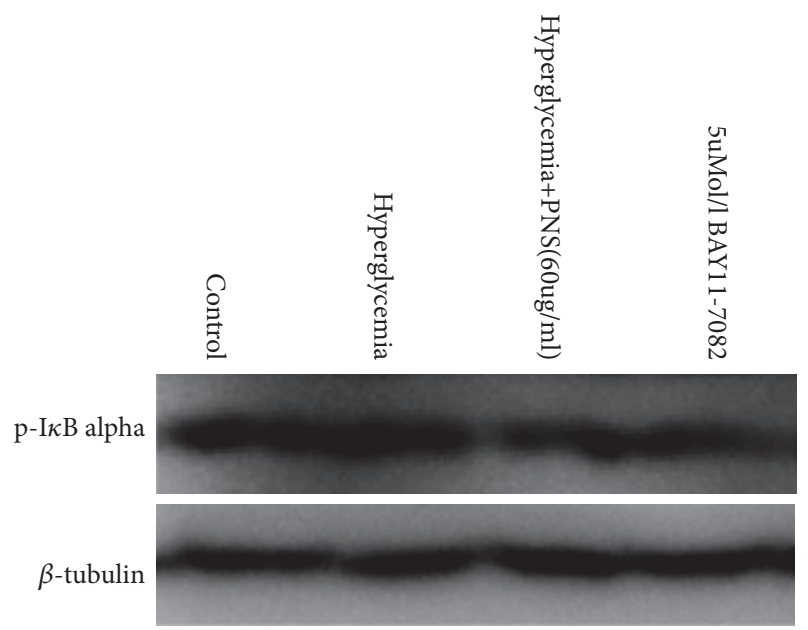

(a)

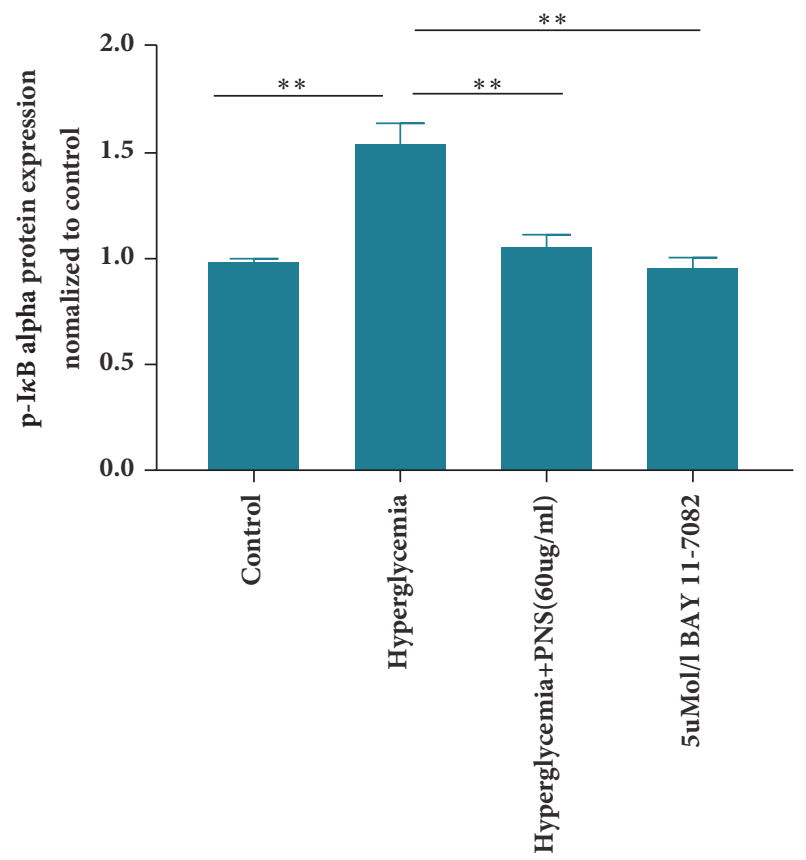

(b)

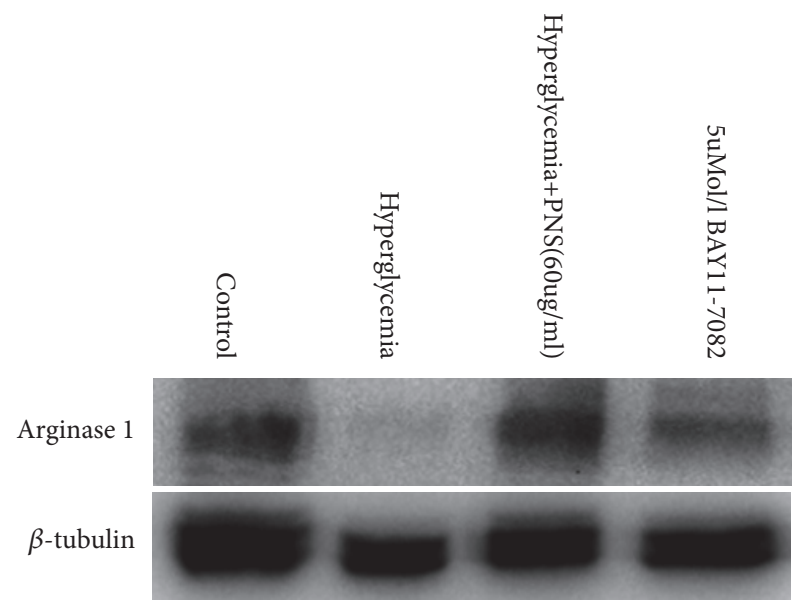

(c)

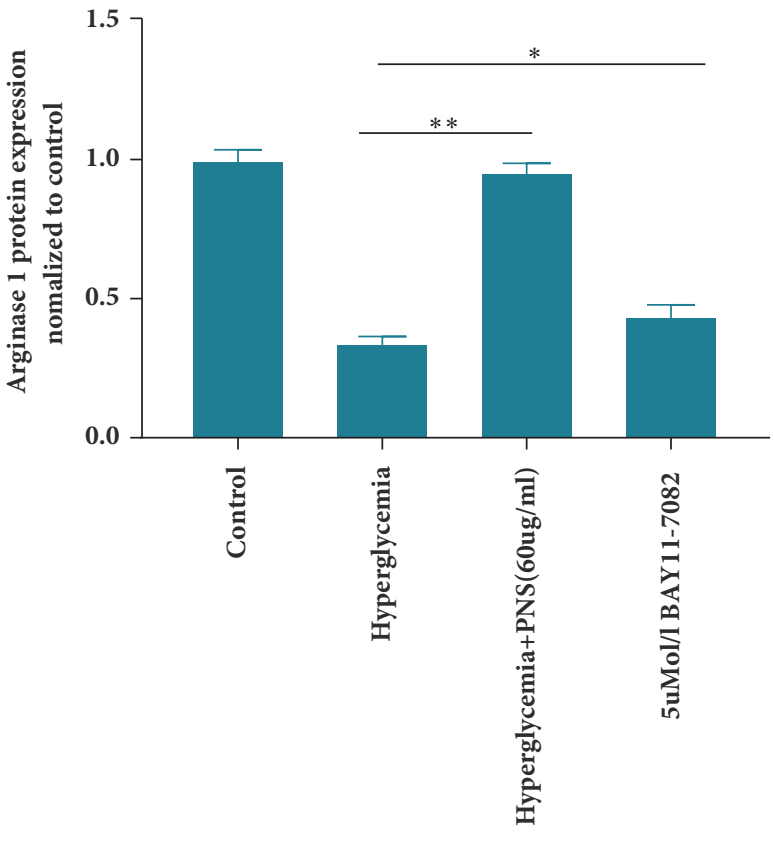

(d)

FIGURE 4: PNS prompt arginase 1 expression by decreasing $\mathrm{I} \kappa \mathrm{B}$-alpha phosphorylation in macrophages under hyperglycemic conditions. THP-1 macrophages were pretreated with 5uM/l BAY 11-7082 for 2 hours before they were stimulated with $15 \mathrm{mMol} / \mathrm{l}$ glucose for 24 hours. In this experiment, cell culture time for PNS group is also 24 hours. Effects of $60 \mathrm{ug} / \mathrm{ml}$ PNS and $5 \mathrm{uM} / 1 \mathrm{BAY} 11-7082$ on I $\kappa \mathrm{B}$-alpha phosphorylation $(\mathrm{a}, \mathrm{c})$ and arginase 1 expression $(\mathrm{b}, \mathrm{d})$ were determined by Western blot ( $\mathrm{n}=3$ per group). Results are expressed as the mean \pm SE. $*$ indicates $p<0.05$ and $* *$ indicates $p<0.01$.

\section{Acknowledgments}

This work was supported by Traditional Chinese Medicine Administration of Zhejiang Province (Project code: 2013ZQ003), Zhejiang Medical Project of Science and Technology (Project code: 2014KYA026), Zhejiang Province Key Subject of Medicine (Project code: 2015-ZDCXXK01-2), and Natural Science Foundation of Zhejiang Province (Project code: LY15H020006).

\section{References}

[1] Y. V. Bobryshev, E. A. Ivanova, D. A. Chistiakov, N. G. Nikiforov, and A. N. Orekhov, "Macrophages and their role in atherosclerosis: pathophysiology and transcriptome analysis," BioMed Research International, vol. 2016, Article ID 9582430, 13 pages, 2016.

[2] A. Mantovani, C. Garlanda, and M. Locati, "Macrophage diversity and polarization in atherosclerosis: a question of balance," 
Arteriosclerosis, Thrombosis, and Vascular Biology, vol. 29, no. 10, pp. 1419-1423, 2009.

[3] C. Poitou, E. Dalmas, M. Renovato et al., "CD14dimCD16+ and CD14+CD16+ monocytes in obesity and during weight loss: relationships with fat mass and subclinical atherosclerosis," Arteriosclerosis, Thrombosis, and Vascular Biology, vol. 31, no. 10, pp. 2322-2330, 2011.

[4] J. E. Lim, E. Chung, and Y. Son, "A neuropeptide, Substance$\mathrm{P}$, directly induces tissue-repairing M2 like macrophages by activating the $\mathrm{PI} 3 \mathrm{~K} / \mathrm{Akt} / \mathrm{mTOR}$ pathway even in the presence of IFNgamma," Scientific Reports, vol. 7, no. 1, p. 9417, 2017.

[5] M. Peled and E. A. Fisher, "Dynamic aspects of macrophage polarization during atherosclerosis progression and regression," Frontiers in Immunology, vol. 5, p. 579, 2014.

[6] A. Mantovani, S. Sozzani, M. Locati, P. Allavena, and A. Sica, "Macrophage polarization: tumor-associated macrophages as a paradigm for polarized M2 mononuclear phagocytes," Trends in Immunology, vol. 23, no. 11, pp. 549-555, 2002.

[7] A. Mantovani, A. Sica, S. Sozzani, P. Allavena, A. Vecchi, and M. Locati, "The chemokine system in diverse forms of macrophage activation and polarization," Trends in Immunology, vol. 25, no. 12, pp. 677-686, 2004.

[8] D. M. Mosser, "The many faces of macrophage activation," Journal of Leukocyte Biology, vol. 73, no. 2, pp. 209-212, 2003.

[9] Y. Zhao, Y. Guo, Y. Jiang, X. Zhu, Y. Liu, and X. Zhang, "Mitophagy regulates macrophage phenotype in diabetic nephropathy rats," Biochemical and Biophysical Research Communications, vol. 494, no. 1-2, pp. 42-50, 2017.

[10] Z. Rao, J. Sun, X. Pan et al., "Hyperglycemia aggravates hepatic ischemia and reperfusion injury by inhibiting liver-resident macrophage M2 polarization via C/EBP homologous proteinmediated endoplasmic reticulum stress," Frontiers in Immunology, vol. 8, p. 1299, 2017.

[11] N. Liu, D. Shan, Y. Li, H. Chen, Y. Gao, and Y. Huang, "Panax notoginseng saponins attenuate phenotype switching of vascular smooth muscle cells induced by notch 3 silencing," Evidence-Based Complementary and Alternative Medicine, vol. 2015, Article ID 162145, 2015.

[12] Q. Shen, J. Li, C. Zhang et al., "Panax notoginseng saponins reduce high-risk factors for thrombosis through peroxisome proliferator-activated receptor-gamma pathway," Biomedicine \& Pharmacotherapy, vol. 96, pp. 1163-1169, 2017.

[13] L. Dou, Y. Lu, T. Shen et al., "Panax notogingseng saponins suppress RAGE/MAPK signaling and NF-kappaB activation in apolipoprotein-E-deficient atherosclerosis-prone mice," Cellular Physiology and Biochemistry, vol. 29, no. 5-6, pp. 875-882, 2012.

[14] K. Kitamura, Y. Takamura, T. Iwamoto et al., "Dammaranetype triterpene extracts of Panax notoginseng root ameliorates hyperglycemia and insulin sensitivity by enhancing glucose uptake in skeletal muscle," Bioscience, Biotechnology, and Biochemistry, vol. 81, no. 2, pp. 335-342, 2017.

[15] N. Leitinger and I. G. Schulman, "Phenotypic polarization of macrophages in atherosclerosis," Arteriosclerosis, Thrombosis, and Vascular Biology, vol. 33, no. 6, pp. 1120-1126, 2013.

[16] M. de Gaetano, D. Crean, M. Barry, and O. Belton, "M1- and M2type macrophage responses are predictive of adverse outcomes in human atherosclerosis," Frontiers in Immunology, vol. 7, p. 275, 2016.

[17] H. J. Williams, E. A. Fisher, and D. R. Greaves, "Macrophage differentiation and function in atherosclerosis: opportunities for therapeutic intervention?" Journal of Innate Immunity, vol. 4, no. 5-6, pp. 498-508, 2012.

[18] P. R. Nagareddy, A. J. Murphy, R. A. Stirzaker et al., "Hyperglycemia promotes myelopoiesis and impairs the resolution of atherosclerosis," Cell Metabolism, vol. 17, no. 5, pp. 695-708, 2013.

[19] H. Hu, H. Jiang, H. Ren, X. Hu, X. Wang, and C. Han, "AGEs and chronic subclinical inflammation in diabetes: Disorders of immune system," Diabetes/Metabolism Research and Reviews, vol. 31, no. 2, pp. 127-137, 2015.

[20] S.-I. Yamagishi, K. Nakamura, and T. Matsui, "Regulation of advanced glycation end product (AGE)-receptor (RAGE) system by PPAR-gamma agonists and its implication in cardiovascular disease," Pharmacological Research, vol. 60, no. 3, pp. 174-178, 2009.

[21] N. Ohgami, A. Miyazaki, M. Sakai, A. Kuniyasu, H. Nakayama, and S. Horiuchi, "Advanced glycation end products (AGE) inhibit scavenger receptor class B type I-mediated reverse cholesterol transport: a new crossroad of AGE to cholesterol metabolism," Journal of Atherosclerosis and Thrombosis, vol. 10, no. 1, pp. 1-6, 2003.

[22] X. Jin, L. Liu, Z. Zhou, J. Ge, T. Yao, and C. Shen, "Pioglitazone alleviates inflammation in diabetic mice fed a high-fat diet via inhibiting advanced glycation end-product-induced classical macrophage activation," FEBS Journal, vol. 283, no. 12, pp. 22952308, 2016.

[23] S. Parathath, L. Grauer, L.-S. Huang et al., "Diabetes adversely affects macrophages during atherosclerotic plaque regression in mice," Diabetes, vol. 60, no. 6, pp. 1759-1769, 2011.

[24] X.-S. Zeng, J.-J. Jia, and L.-F. Ma, "Gensenoside Rb1 protects rat PC12 cells from oxidative stress-induced endoplasmic reticulum stress: the involvement of thioredoxin-1," Molecular and Cellular Biochemistry, vol. 410, no. 1-2, pp. 239-246, 2015.

[25] Y. Jia, Z.-Y. Li, H.-G. Zhang, H.-B. Li, Y. Liu, and X.-H. Li, "Panax notoginseng saponins decrease cholesterol ester via upregulating ATP-binding cassette transporter A1 in foam cells," Journal of Ethnopharmacology, vol. 132, no. 1, pp. 297-302, 2010.

[26] C.-Y. Yang, J. Wang, Y. Zhao et al., "Anti-diabetic effects of Panax notoginseng saponins and its major anti-hyperglycemic components," Journal of Ethnopharmacology, vol. 130, no. 2, pp. 231-236, 2010.

[27] A. Rhule, B. Rase, J. R. Smith, and D. M. Shepherd, “Toll-like receptor ligand-induced activation of murine DC2.4 cells is attenuated by Panax notoginseng," Journal of Ethnopharmacology, vol. 116, no. 1, pp. 179-186, 2008.

[28] J. Zhang, L. Ding, B. Wang et al., "Notoginsenoside R1 Attenuates Experimental Inflammatory Bowel Disease via Pregnane $\mathrm{X}$ Receptor Activation," The Journal of Pharmacology and Experimental Therapeutics, vol. 352, no. 2, pp. 315-324, 2014.

[29] B. Shu, Y. Feng, Y. Gui et al., "Blockade of CD38 diminishes lipopolysaccharide-induced macrophage classical activation and acute kidney injury involving NF- $\kappa \mathrm{B}$ signaling suppression," Cellular Signalling, vol. 42, pp. 249-258, 2018.

[30] Y. Wang, Z. Song, J. Bi et al., "A20 protein regulates lipopolysaccharide-induced acute lung injury by downregulation of NF- $\kappa \mathrm{B}$ and macrophage polarization in rats," Molecular Medicine Reports, vol. 16, no. 4, pp. 4964-4972, 2017.

[31] Q. Zhang, M. J. Lenardo, and D. Baltimore, "30 Years of NF- $\kappa$ B: A Blossoming of Relevance to Human Pathobiology," Cell, vol. 168, no. 1-2, pp. 37-57, 2017. 


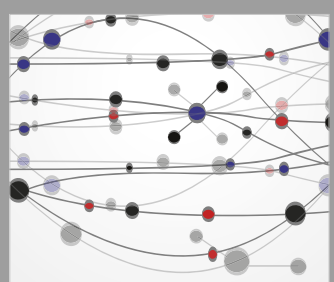

The Scientific World Journal
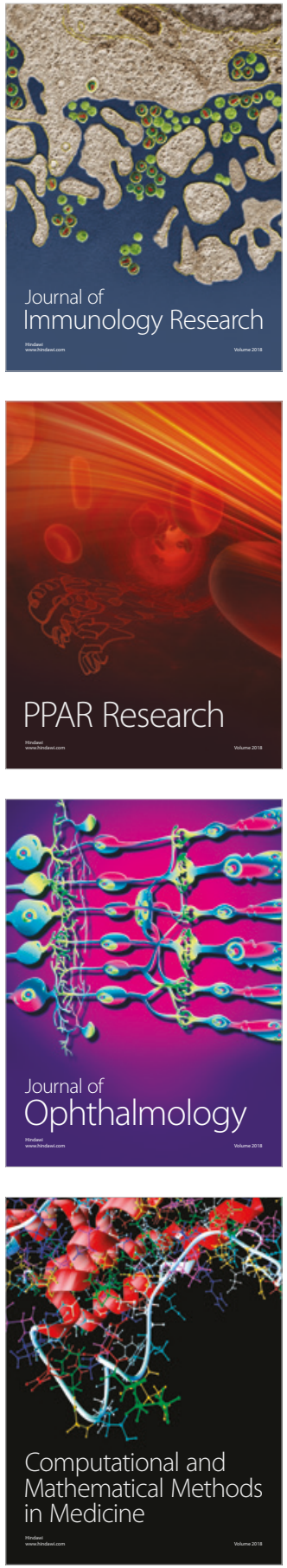

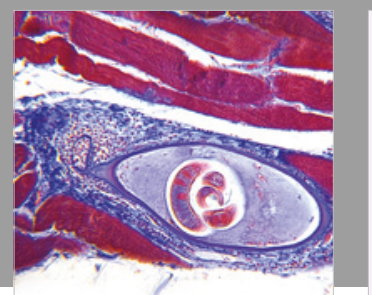

Gastroenterology Research and Practice

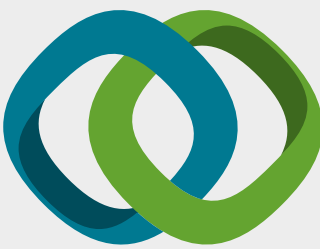

\section{Hindawi}

Submit your manuscripts at

www.hindawi.com
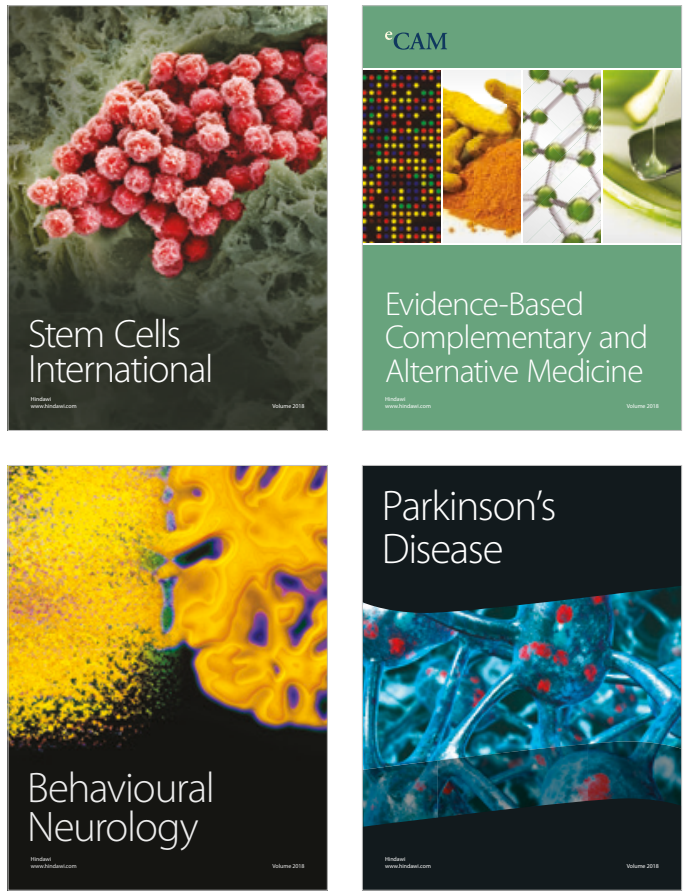

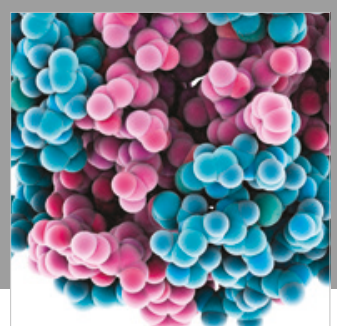

ournal of

Diabetes Research

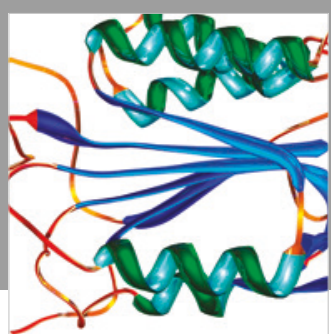

Disease Markers
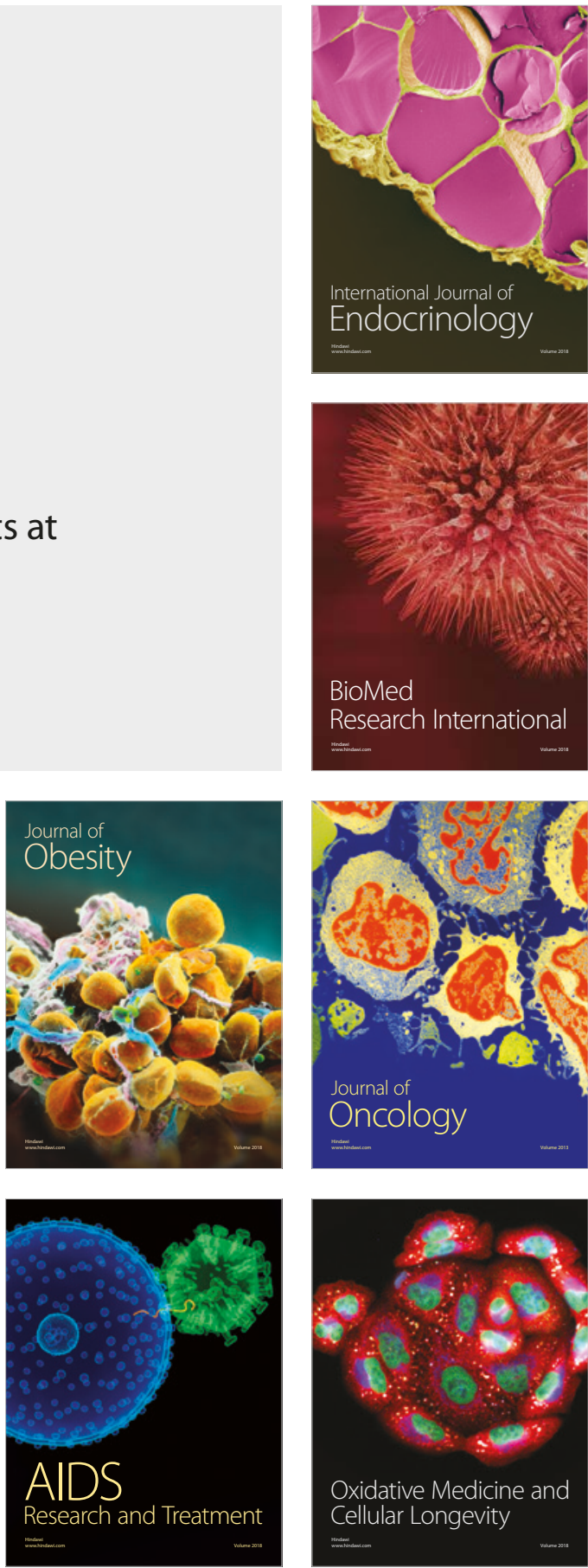